\title{
RNA-Seq reveals the existence of a CDKN1C-E2F1-TP53 axis that is altered in human T-cell lymphoblastic lymphomas
}

\author{
Pilar López-Nieva ${ }^{1,2,3+}$, Pablo Fernández-Navarro ${ }^{4,5 \dagger}$, Concepción Vaquero-Lorenzo ${ }^{1 \dagger}$, María Villa-Morales ${ }^{1,2,3}$, \\ Osvaldo Graña-Castro ${ }^{6}$, María Ángeles Cobos-Fernández ${ }^{1,2}$, José Luis López-Lorenzo², Pilar Llamas², \\ Laura González-Sanchez ${ }^{1,2,3}$, Isabel Sastre ${ }^{1}$, Marina Pollan ${ }^{4,5}$, Marcos Malumbres ${ }^{7}$, Javier Santos ${ }^{1,2,3^{*}}$ \\ and José Fernández-Piqueras ${ }^{1,2,3^{*}}$ (D)
}

\begin{abstract}
Background: Precursor T-cell lymphoblastic lymphomas (T-LBL) are rare aggressive hematological malignancies that mainly develop in children. As in other cancers, the loss of cell cycle control plays a prominent role in the pathogenesis in these malignancies that is primarily attributed to loss of CDKN2A (encoding protein p16INK4A). However, the impact of the deregulation of other genes such as CDKN1C, E2F1, and TP53 remains to be clarified. Interestingly, experiments in mouse models have proven that conditional T-cell specific deletion of Cdkn1c gene may induce a differentiation block at the DN3 to DN4 transition, and that the loss of this gene in the absence of Tp53 led to aggressive thymic lymphomas.

Results: In this manuscript, we demonstrated that the simultaneous deregulation of CDKN1C, E2F1, and TP53 genes by epigenetic mechanisms and/or the deregulation of specific microRNAs, together with additional impairing of TP53 function by the expression of dominant-negative isoforms are common features in primary human T-LBLs.

Conclusions: Previous experimental work in mice revealed that T-cell specific deletion of $\mathrm{Cdkn1c}$ accelerates lymphomagenesis in the absence of Tp53. If, as expected, the consequences of the deregulation of the CDKN1CE2F1-TP53 axis were the same as those experimentally demonstrated in mouse models, the disruption of this axis might be useful to predict tumor aggressiveness, and to provide the basis towards the development of potential therapeutic strategiesin human T-LBL.
\end{abstract}

Keywords: T-cell lymphoblastic lymphoma, CDKN1C-E2F1-TP53 deregulation, Promoter hypermethylation, Deregulation of miRNAs

\section{Background}

Precursor T-cell lymphoblastic neoplasms are aggressive haematological malignancies that mainly develop in children (in particular adolescent males) but also in adults. They derive from maturing thymocytes leading to excessive lymphoblastoid cells in the bone marrow and other lymphoid organs. Clinically, T-cell acute lymphoblastic leukaemia (T-ALL) and T-cell lymphoblastic lymphoma

\footnotetext{
*Correspondence: javier.santos@uam.es; jfpiqueras@cbm.csic.es

${ }^{\dagger}$ Equal contributors

${ }^{1}$ Department of Cellular Biology and Immunology, Severo Ochoa Molecular Biology Center (CBMSO), CSIC-Madrid Autonomous University, 28049 Madrid, Spain

Full list of author information is available at the end of the article
}

(T-LBL) are two subgroups differing by the extent of bone marrow infiltration. T-ALL manifests with extensive bone marrow and blood affectation, whereas a mass lesion in the thymus/anterior mediastinum with less than $25 \%$ of lymphoblasts in the bone marrow characterizes T-LBL [1].

As in other cancers, the loss of cell cycle control plays a prominent role in the pathogenesis of these malignancies that is primarily attributed to loss of CDKN2A (which encodes the tumour suppressor protein p16INK4A) and, to a lesser extent, loss of $R B 1$ or $C D K N 1 B$ (which encodes $\mathrm{p} 27 / \mathrm{KIP} 1$ protein) and aberrantly high levels of CCND2 (encoding cyclin D2) [2]. 
Downregulation of CDKN1C (which encodes p57/KIP2 protein) by promoter hypermethylation has been detected with very low frequency in paediatric T-ALL and more often in adult patients. However, the biological and clinical impact of hypermethylation and/or loss of CDKN1C expression remain uncertain [3]. In addition to T-ALL, downregulation of CDKN1C has been observed more frequently in a wide variety of human tumours associated with a strengthening of cell proliferation $[4,5]$.

In addition, numerous studies have reported that $E 2 F 1$ overexpression has clinical relevance in many types of cancers [6]. However, to the best of our knowledge, E2F1 alterations have not been so far implicated in the development of precursor T-cell neoplasms.

Moreover, the gene encoding TP53 protein, a main downstream effector of E2F1, is frequently targeted in human tumours by gene mutations [7, 8]. Apart from the canonical full-length transcript, it should be noted that alternative splicing of TP53 and the use of alternate promoter might result in multiple transcript variants and isoforms [9] and, interestingly, abnormal expression of TP53 isoforms has been reported in many cancers as head and neck, acute myeloid leukaemia (AML) and breast tumours [10] but not in T-cell lymphoblastic neoplasms.

The potential nexus between these three genes has been demonstrated in mice. Some authors [11] have shown in mouse models that inactivation of the Cdkn1c gene (also termed as $p 57^{K I P 2}$ ) results in thymocyte development arrest at DN3 (Double-Negative 3) to DN4 cells transition, due to hyper-activation of the E2f-Tp53 pathway. Furthermore, the loss of $C d k n 1 c$ accelerates the development of thymic lymphomas in the absence of the $T p 53$ gene.

To assess whether the axis CDKN1C/E2F1/TP53 plays a role in human T-cell lymphoblastic lymphomas, we investigated the mutational status and the expression levels of these three genes using Next-Generation Sequencing (NSG) approaches. Interestingly, RNASequencing analysis revealed reduced levels of $C D K N 1 C$ mRNA in almost all analysed T-LBL samples, which may be accompanied by increased expression of E2F1 and overexpression of the TP53 transcript variant encoding the $\triangle 133 T P 53$ isoform. Deregulation of these genes is executed by epigenetics mechanisms and deregulation of specific miRNAs.

\section{Methods}

\section{Human sample collection}

Human T-LBL samples separated in an exploratory cohort (8 samples), an extended cohort (10 samples), and four thymuses of human foetus without haematological pathology, were obtained from the Spanish Hospital
Biobanks Network (RetBioH; www.redbiobancos.es). Lymphomas were diagnosed according to World Health Organization Classification of Hematological Malignancies and recommendations from the European childhood lymphoma pathology panel [12, 13] (Additional file 1: Table S1). Institutional review board approval was obtained for these studies (reference CEI:70-1260).

\section{RNA-sequencing}

Total RNA was obtained using TriPure Reagent (Roche Applied Science, Indianapolis, IN, USA), following manufacturer's instructions.

\section{Massive sequencing of $m R N A s$}

RNA Integrity Numbers (RIN) were in the range of 7.2-9.8. Image analysis, per-cycle basecalling and quality score assignment were performed with Illumina Real Time Analysis software (Illumina, San Diego, CA). BCL files were converted to FASTQ format with Illumina's Off-Line Basecaller package (Illumina). The resulting directional RNA-seq libraries were sequenced in paired-end format in two different rounds (Illumina HiSeq2000), leading to $50 \mathrm{bp}$ and $76 \mathrm{bp}$ reads (the latter were trimmed to $50 \mathrm{bp}$ ). Sequenced reads were quality-checked with FastQC (http://www.bio informatics.babraham.ac.uk/projects/fastqc/). RNA-seq reads were aligned to the human genome (GRCh37/hg19) with TopHat-2.0.10 [14] (using Bowtie 1.0.0 [15] and Samtools 0.1.19 [16]) allowing two mismatches and five multihits. Transcripts assemblies, estimation of their abundances were calculated with Cufflinks 2.2.1, using the Ensembl GRCh37.74 annotation for human. In this analysis, we only considered the transcripts isoforms of the genes CDKN1C, E2F1 and TP53 that encode for proteins according to the information showed in Ensembl [17].

\section{Small RNA}

Image analysis and per-cycle basecalling was performed with Illumina Real Time Analysis software (RTA1.9) (Illumina). Conversion to FASTQ read format was performed by CASAVA-1.8 (Illumina). Small-RNA-seq libraries were sequenced as 40 bp single-end reads (Illumina Genome Analyzer IIx, GAIIx). Sequenced reads were quality-checked with FastQC. Sequence adapters were removed with cutadapt v1.2.1 [18] and only those reads longer than $15 \mathrm{bp}$ and shorter than $35 \mathrm{bp}$ were kept for further analysis. Reads were aligned to the human genome (GRCh37/hg19) with Bowtie 1.0.0 [15] and Samtools 0.1.19 [16] allowing no mismatches and a maximum of one alignment per read. Raw counts for miRNAs were obtained with HTSeq v0.5.3p9 [19], using the miRBase v20 [20] annotation for hg19. A table with normalized read counts was generated with DESeq [21] and was used to filter out miRNAs with 
questionable expression and outliers. The following criteria were used: first, we required that a miRNA should have a minimal normalized count value of 15 in at least $5 \%$ of the samples. Second, miRNAs with normalized expression values across the samples that exceeded Q13*IQR or Q3 + 3*IQR were considered outliers and discarded. For the remaining miRNAs, $\log 2$ fold-changes of expression were calculated.

Raw sequencing data and transcripts expression quantification is available as a superseries in GEO (Gene Expression Omnibus) under the following ID: GSE109234.

\section{Additional criteria to select miRNAs}

To select those miRNA controlling CDKN1C, E2F1 and TP53 genes, we used the databases of miRGate and miRTarBase. We select those miRNAs experimentally validated ("Functional miRNA-target interactions (MTI)" registered in "Support type" of miRTarBase and/or "Functional MTI" registered in the miRGate "Confirmed predictions") and/or those microRNA that showed a "miRGate Agreement Score" equal or higher than the median agreement-value of the microRNA identified associated with the genes assessed (median value $=1.04$ ). (Additional file 2: Figure S1).

Additionally, we filtered out miRNAs showing a number of counts lower than 28.70 (median value of the miRNA counts of all the samples) in any sample (Additional file 3: Figure S2).

\section{Quantitative RT-PCR}

RNA was reverse-transcribed using first the HighCapacity RNA-to-cDNA ${ }^{\text {ma }}$ Kit (Applied Biosystems, Foster City, CA, USA) and MystiCq microRNA cDNA Synthesis Mix (Sigma-Aldrich, St. Louis, MO, USA). Quantitative real-time PCR reactions were performed in triplicate with an Applied Biosystems 7300 RealTime PCR system (Life Technologies, Carlsbad, CA), using either the Fast Start Universal SYBRGreen Master (Rox) (Roche) or the MystiCq microRNA SYBR Green qPCR ReadyMix (Sigma-Aldrich), according to the manufacturers' instructions. Expression values of $\beta$-2-microglobulin or $\beta$-actin or SNORD48 served to normalize using the 2- $\Delta \Delta \mathrm{C} \mathrm{T}$ method [22]. Primers are indicated in Additional file 4: Table S2.

\section{Targeted gene deep sequencing and sanger sequencing} Mutational status of CDKN1C, E2F1 and TP53 genes was analysed by targeted deep sequencing in genomic using a selected panel of cancer-related genes (the OncoNIM $^{\circ}$ Seq409 panel; New Integrated Medical genetics; NIMGenetics, Madrid, Spain). Sanger DNA sequencing of PCR-amplified mutational hot spots was performed with the specific primers summarized in Additional file 4: Table S2.
Bisulfite genomic sequencing and methylation-specific PCR (MSP)

Methyl Primer Express v1.0 software (Applied Biosystems) was used to identify $\mathrm{CpG}$ islands around the Transcriptional Star Site (TSS) of CDKN1C gene, and to design specific primers for the methylation analysis. DNA $(1 \mu \mathrm{g})$ was subjected to sodium bisulfite treatment using the EZ DNA Methylation-Gold kit (Zymo Research, CA, USA). MSP was performed with primers specific for methylated (M) or unmethylated (U) CpG sites. For bisulfite genomic sequencing, a region included in the one analysed by MSP was amplified using $1 \mu \mathrm{L}$ of bisulfite-converted DNA with Immolase Taq polymerase (Bioline USA Inc., Kenilworth, $\mathrm{NJ})$ at $60{ }^{\circ} \mathrm{C}$ for 40 cycles. Then the resulting PCR products were gel-purified (2\% agarose) with Wizard ${ }^{\circledR} \mathrm{SV}$ Gel and PCR Clean-Up System (Promega, Madison, WI, USA) and cloned into the pGEMT Easy Vector System (Promega) following the manufacturer-specific protocols. For all samples, 12 colonies were randomly chosen, and DNA was purified using Wizard ${ }^{\circledR}$ Plus SV Minipreps DNA Purification System (Promega) and sequenced with a ABI 3730 xl DNA Analyzer (Applied Biosystems). After sequencing analysis, the results were transformed into percentages of CpGs calculated in comparison with the total CpGs of the analysed region. Primers and conditions are indicated in Additional file 4: Table S2.

\section{Statistical analyses}

Differential expression of mRNA and miRNA (RNASeq) between tumours and controls was estimated by calculating the $\log 2$ Fold changes $(\log 2 \mathrm{FC})$ of the expression levels. Only differential expression levels estimated by the Cufflinks software as "OK" were taken into account. Significant deregulated miRNAs with $\log 2 \mathrm{FC}$ absolute values equal or higher than 1.5 in at least in one sample were selected according to the information of miRGate and miRTarBase databases [23, 24] and additional criteria based on the read counts [25]. Student's t-test was used to compare results from qRT-PCR between tumours and controls. All statistical analyses were performed using $\mathrm{R}$ software.

\section{Results}

\section{Deregulation of CDKN1C, E2F1 and TP53 in T-LBLs}

The results of massive RNA-sequencing (RNA-Seq) of the transcript isoforms that encode proteins in the $8 \mathrm{~T}$ LBL samples of the exploratory cohort showed that the mRNA level of CDKN1C was strongly reduced in all analysed tumours compared to that of the normal foetal thymuses, with fold-changes ranging from -25.99 to 2.15 in the canonical isoform ENST00000414822. By contrary, the expression of E2F1 gene in the same panel increases in all tumours, three of them with foldchanges higher than 1.5. Concerning the transcriptional 
status of the TP53 gene, we found increased levels of the transcript variant encoding the dominant-negative $\Delta 133$ p53 $\alpha$ protein isoform (TP53-008: ENST00000504937) in all but two of the eight tumours (fold-changes between 3.26 and 0.74). Moreover, two of them (346 and 460) exhibited a clear reduction of two transcript variants (TP53-001: ENST00000269305 and TP53-002: ENST00000445888) encoding full-length TP53 protein isoforms (TAp53 $\alpha$ ). Finally, two tumours (192 and 521) showed increased amounts of the TP53 $\beta$ transcript (ENST00000420246), which encodes a C-terminal truncated protein (Figs. 1 and 2; Additional file 5: Table S3).

These results were validated by quantitative real-time RT-PCR (qRT-PCR) analysis and confirmed in the extended cohort (Fig. 3; Additional file 6: Table S4). Interestingly, all samples in the extended cohort showed a significant reduction of CDKN1C expression, six out of ten showed significant increases of $E 2 F 1$ expression, and six out of ten exhibited significant increases of the mRNA isoform encoding $\Delta 133 \mathrm{p} 53 \alpha$ protein.

\section{Overrepresentation of the arginine allele at codon 72 of TP53 in T-LBLs}

The analysis of T-LBLs by targeted gene deep sequencing revealed the existence of two missense mutations. One of them was c.427G > T (p.Val143Leu) at exon 5 in sample 192, with conflicting interpretations of pathogenicity in the IARC database [26]. The other missense mutation was the functional polymorphism c. $215 \mathrm{C}>\mathrm{G}$ (p.Pro72Arg) that was found in all but one analysed tumours (8/9), three of them being homozygotes for the arginine allele (238, 521, and 840) (Additional file 7 : Table S5) (Fig. 2). However, we were able to validate only the mutation at exon 4 by DNA Sanger-sequencing (data not shown) using the primers and conditions indicated in Additional file 4: Table S2.

\section{Epigenetic modifications contribute to the altered expression of CDKN1C in a fraction of T-LBLs}

The CDKN1C gene is remarkably rich in CpG islands situated both upstream and downstream from the transcriptional start site, whose hypermethylation has been strongly related to its inactivation [27]. To elucidate whether aberrant DNA methylation is a mechanism whereby CDKN1C was downregulated in our T-LBLs, we analysed the DNA methylation levels of the promoter region of $C D K N 1 C$ gene using the $\mathrm{MSP} / \mathrm{Se}$ quencing method. We initially examined six samples of normal foetal thymuses by MSP, confirming the absence of methylated bands in all cases (data not shown). However, although all samples in the exploratory cohort exhibited significant downregulation of this gene, only two tumours exhibited high levels of hypermethylation (521 and 840), suggesting additional mechanisms to explain CDKN1C downregulation in the remaining samples (Fig. 4).

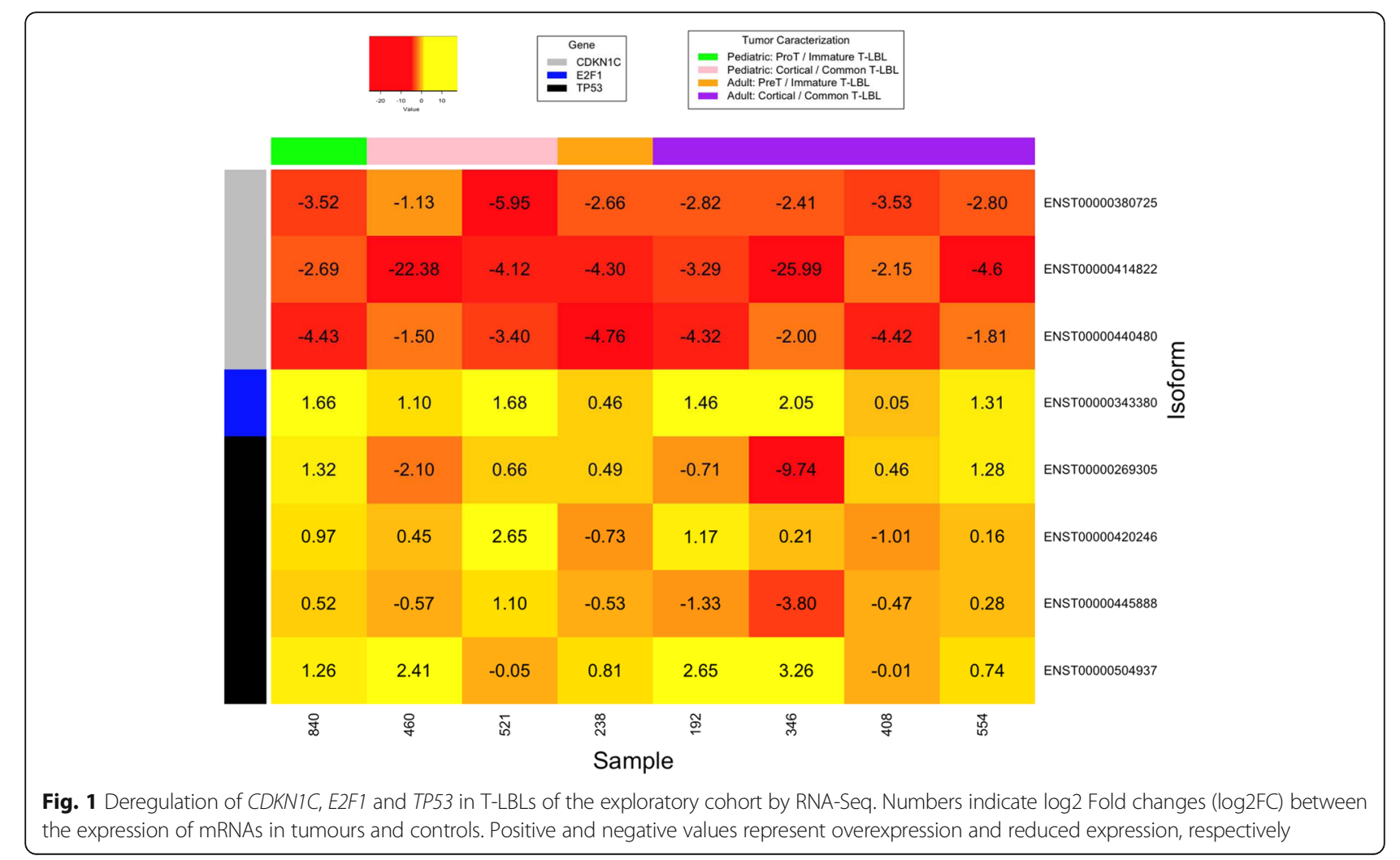


a

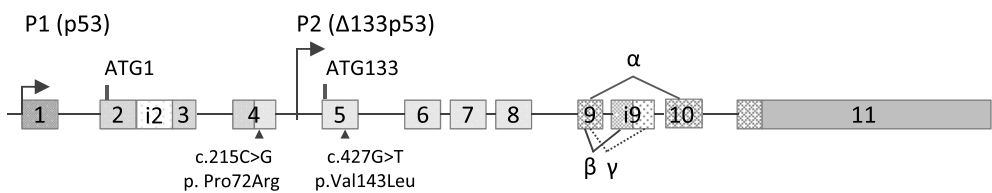

b

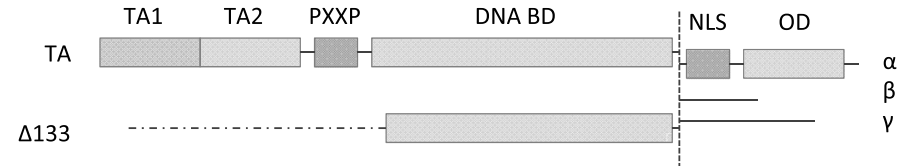

Fig. 2 TP53 mutations and isoforms showing differential expression in our sample series of T-LBL. a Genomic representation of the TP53 gene showing the two missense mutations at exons 4 and 5 we detected in these samples. Alternative splicing of intron 9 generates p53 isoforms bearing different C-terminal domains ( $a, \beta$ and $\gamma$ ). $\mathbf{b}$ TP53 isoforms that showed differential levels of expression in our tumours. TAp53 isoforms include p53 (p53a), p53 $\beta$ and p53 $\gamma$, whereas $\Delta 133 p 53$ isoforms include $\Delta 133 p 53 a, \Delta 133 p 53 \beta$ and $\Delta 133 p 53 \gamma$. TP53 protein domains: transactivation domains (TA), proline rich domain (PXXP), DNA binding domain (DNA BD), nuclear localization signal (NLS) and oligomerization domain (OD)

MicroRNA deregulation contributes to the deregulation of CDKN1C, E2F1 and TP53 genes in T-TLBLs

Aberrant expression of microRNAs (miRNAs) in human tumours and links between deregulated miRNAs and target genes involved in cell cycle have been well established [28, 29].

Differences in the expression level of the selected miRNA were initially determined by massive small RNA-sequencing (RNA-Seq) in the 8 T-LBL samples of the exploratory cohort. There were only two significant deregulated miRNAs controlling CDKN1C expression, which are up-regulated in practically all the samples (miR-221-3p and miR-222-3p). We also found seven deregulated miRNAs to target $E 2 F 1$ transcript, especially emphasizing the importance of miR-203a and miR-205$5 \mathrm{p}$ that were strongly down-regulated. Finally, $17 \mathrm{miR}$ NAs to target TP53 transcripts were deregulated highlighting the levels of miR-200a-3p and miR-375 downregulation of (Fig. 5; Additional file 8: Table S6).

Deregulation of these miRNAs was confirmed by qRTPCR in the exploratory cohort and in the extended cohort of T-LBL samples (Fig. 6; Additional file 9: Table S7).

\section{Discussion}

It is well established that $C D K N 1 C$ and $E 2 F 1$ are two critical controllers of the cell cycle. The overexpression of CDKN1C may cause cell cycle arrest in human tumour cell lines [30, 31], and this inhibitory effect may be reversed by siRNAs against the CDKN1C gene [32]. In contrast, knockdown of E2F1 by RNA interference impairs proliferation of rat glioma cells [33]. Importantly, previous experimental work in mice reported that conditional T cell-specific deletion of $C d k n 1 c$ gene induced a differentiation block in mouse immature thymocytes that is caused by hyperactivation of E2f1 and Tp53 and may be predisposed to thymic lymphoma development. Moreover, $C d k n 1 c$ ablation led to the development of aggressive thymic lymphomas with a reduced latency in a Tp53-null background. Thus, these results suggested a critical role for the $C d k n 1 c-E 2 f 1-T p 53$ axis in mouse thymic lymphoma development [11, 34].

Our results show that all analysed human T-LBL samples exhibited a strong downregulation of CDKN1C. In addition, most of them also exhibited upregulation of E2F1 (6/8 in the exploratory cohort and 6/10 in the extended cohort), which may be accompanied by impairment of TP53 function in some cases (4/6 in the exploratory cohort and $6 / 10$ in the extended cohort) (Fig. 1; Additional file 3: Table S3 and Additional file 4: Table S4). Thus, our data are consistent with the existence and deregulation of a CDKN1C-E2F1-TP53 axis in human T-LBL. However, it should be noted that our study is largely based on the expression of these genes at the transcriptional level. The relationship between mRNA and protein expression levels is dependent on the combined outcomes of mRNA stability, translation, and protein degradation. Notwithstanding, it has been reported that at least 30 to even $85 \%$ of the variation in protein levels can be attributed to variation in mRNA expression [35]. Other authors [36] reported that differentially expressed mRNAs correlate significantly better with their protein product than non-differentially expressed mRNAs, therefore providing some optimism for the usefulness on inferences from mRNA expression in general.

Concerning the mechanisms by which these genes are deregulated, it is well known that $C D K N 1 C$ is subject to a complex regulation involving the cooperation of a $\mathrm{CpG}$ island at its promoter region and distal regulatory elements, such as the imprinting control region Kv-Differentially 


\section{CDKN1C}

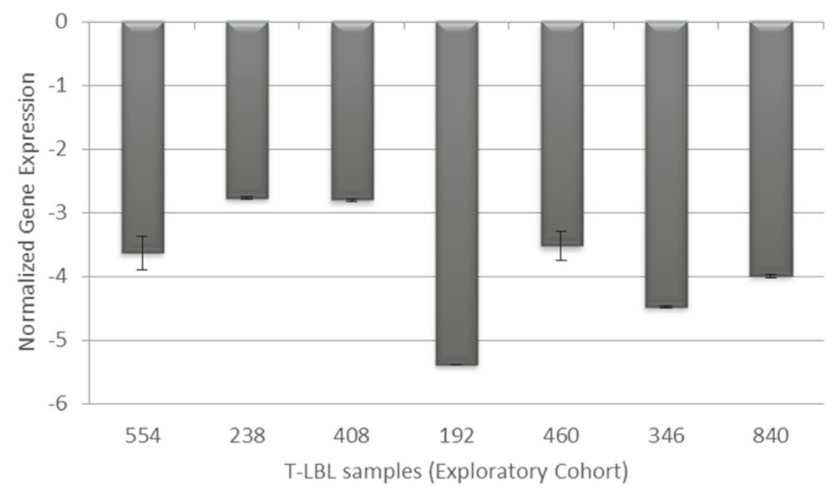

E2F1

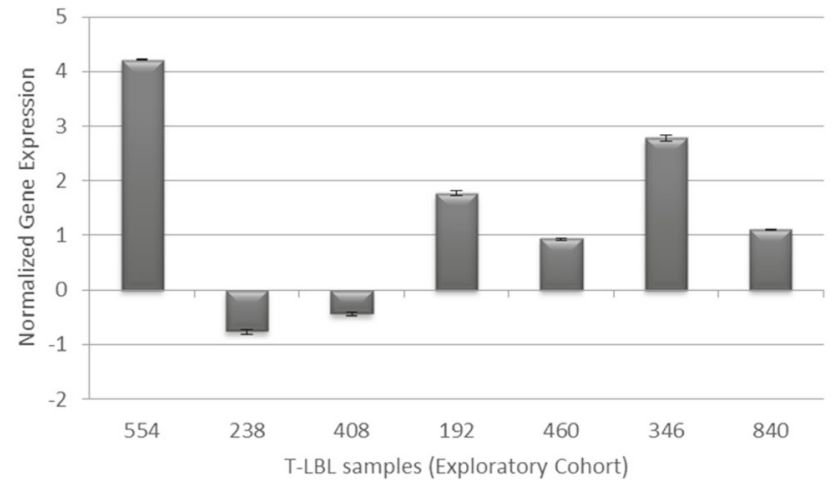

TP53

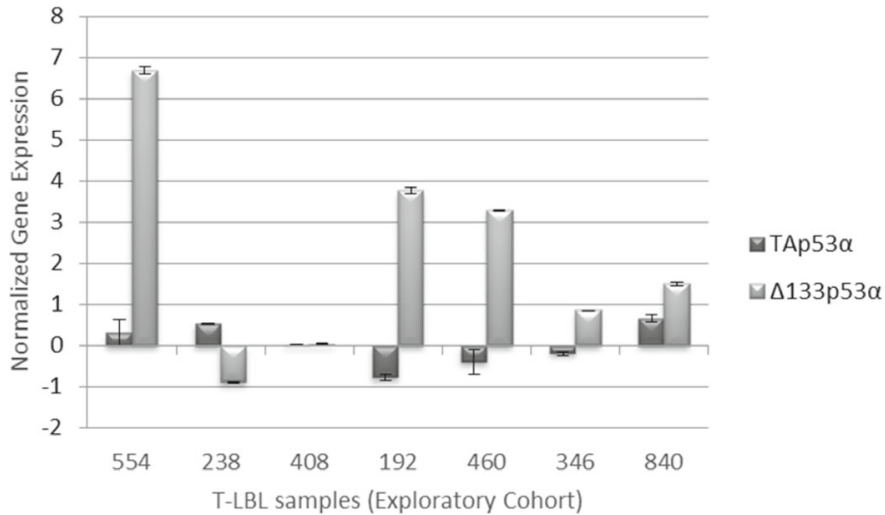

Fig. 3 Differential expression of CDKN1C, E2F1 and TP53 in T-LBLS of the exploratory cohort by quantitative RT-PCR. Relative expression values were calculated as the mRNA amount of each gene relative to that of either $\beta$-actin or $\beta 2$ microglobulin (used as reference) and normalized to the relative expression of normal control samples (foetal thymuses). Each bar represents the mean \pm SD of three independent experiments. Differences in expression values were statistically significant $(p<0.05)$

Methylated Region 1 (KvDMR1) in the promoter of the noncoding KCNQ1OT1 [37, 38]. Although the biological and clinical impact of $C D K N 1 C$ hypermethylation is rather uncertain, aberrant DNA methylation of CDKN1C in its promoter region has been reported in lymphoid malignancies of B and T-cell phenotype [39, 40]. However, $C D K N 1 C$ has been reported downregulated in other type of cancer cells mainly by histone modifications operating in critical regions of its promoter [41, 42]. We initially focused on promoter hypermethylation to explain downregulation of this gene in our sample series of T-LBL, but despite a substantial reduction in the levels of mRNA in almost all samples in the exploratory cohort (7/8), only two samples (840 and 521) (2/8) exhibited significant hypermethylation density (Fig. 4), and six out of eight (including tumor 840 with promoter hypermethylation) exhibited upregulation of one or two miRNAs selected for CDKN1C regulation (miR-211-3p and miR-222-3p). 


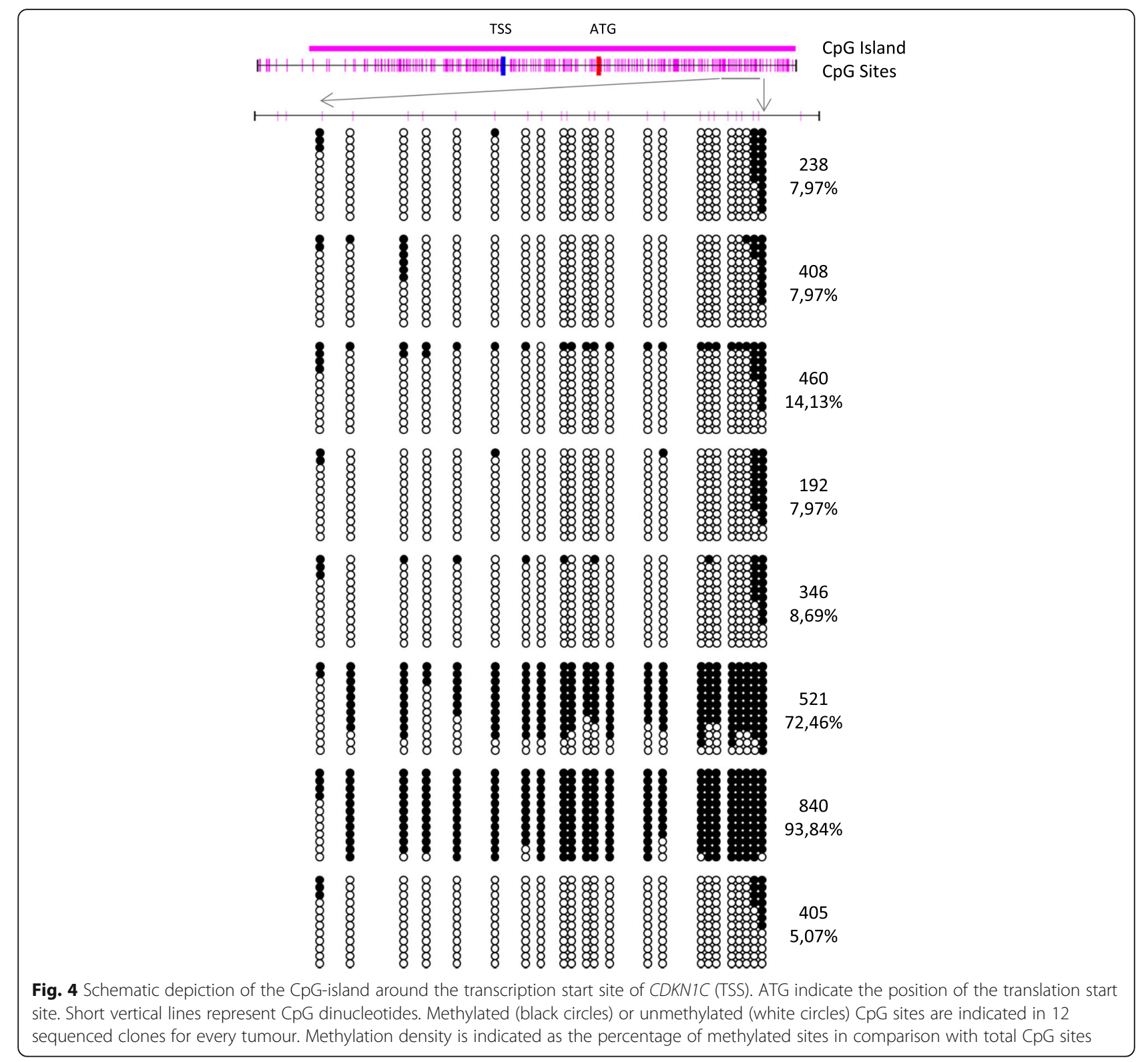

Thus, downregulation of CDKN1C in two samples (33 and 346) should be explained by a different transcriptional mechanism.

Besides this epigenetic mechanism, regulation by miRNAs might be an additional way contributing to determine CDKN1C transcript levels in T-LBLs. Results reported here are in line with those reported in the literature describing miR-25, miR-221 and miR-222 as direct regulators of $C D K N 1 C$ expression in a wide variety of solid tumours, showing a new mechanism responsible for CDKN1C downregulation in carcinogenesis [43-45]. In this context, our findings suggest that aberrant expression of miR-221 and miR-222 may have an oncogenic function in T-LBL development by targeting CDKN1C. However two samples (33 and 346) showed a pronounced downregulation of CDKN1C in the absence of significant changes in miRNA expression (Figs. 5 and 6) or promoter $\mathrm{CpG}$ methylation, thus indicating that the mechanism regulating the expression of this gene is far more complex.

Overexpression of E2F1 may promote proliferation or cell cycle progression by increasing the transcription of genes that contribute to G1-S transition [46]. Notwithstanding at the same time it may also induce apoptosis by multiple pathways, some of which induce stabilization and activation of the TP53 protein [47]. Our microRNA analysis also revealed a consistent deregulation of seven miRNAs in T-LBLs, miR-203a and miR-205-5p being the most representative downregulated microRNAs (Figs. 5 and 6). Interestingly, downregulated miRNAs 


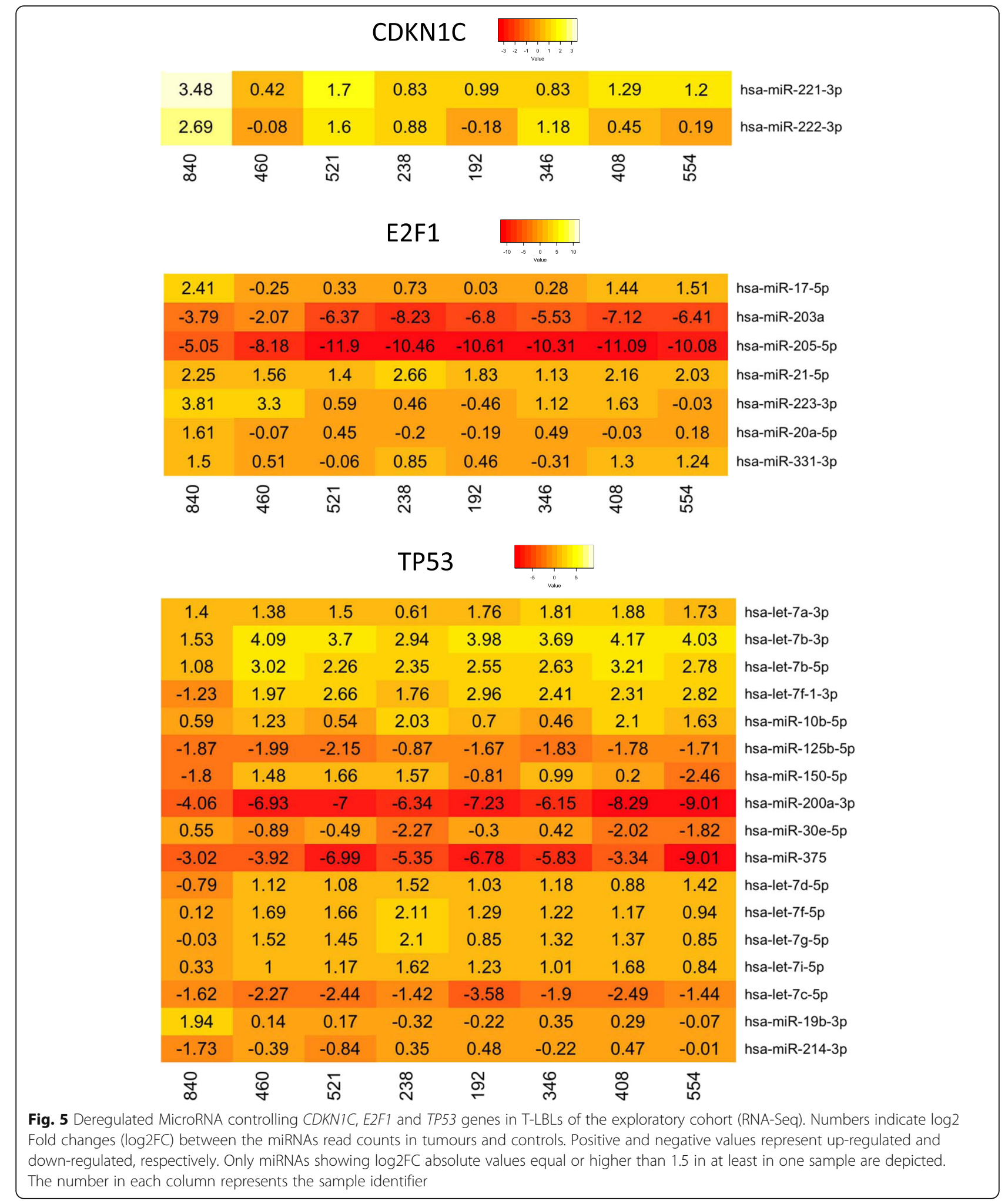

showed higher fold changes than upregulated microRNAs. miR-205-5p is known to be down-regulated in melanoma and its expression inversely correlated with that of $E 2 F 1$ [48].
Concerning impairing of TP53 function, we found overexpression of the human $\Delta 133 p 53 \alpha$ isoform in 4 samples from the exploratory cohort, from which three also exhibited downregulation of the isoform encoding 
hsa-miR-200a-3p

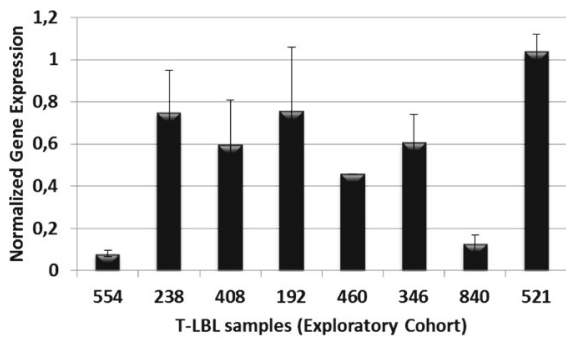

hsa-miR-205-5p

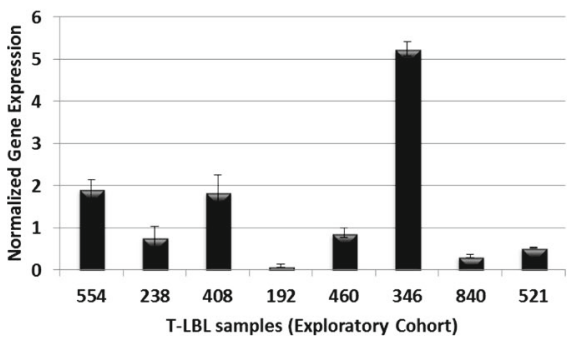

hsa-miR-222-3p

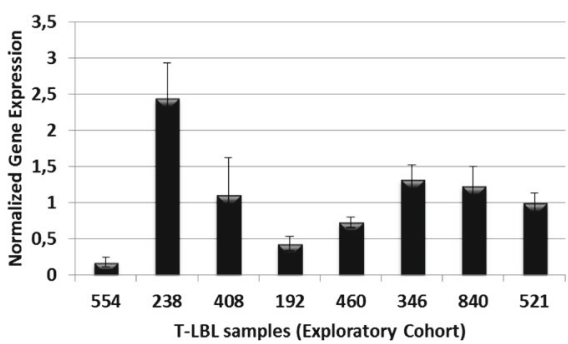

hsa-miR-375

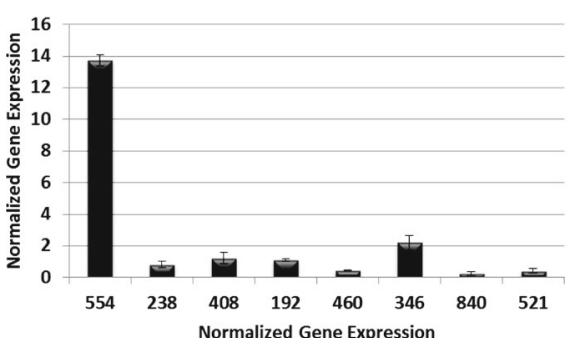

hsa-miR-203

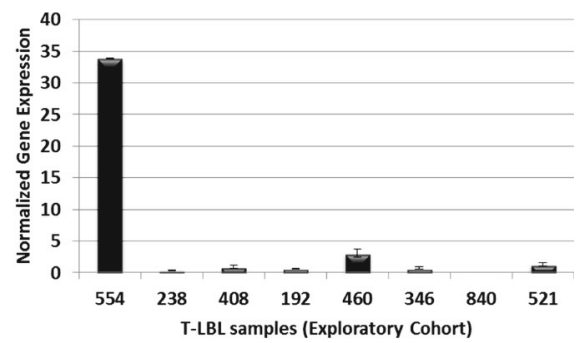

hsa-miR-221-3p

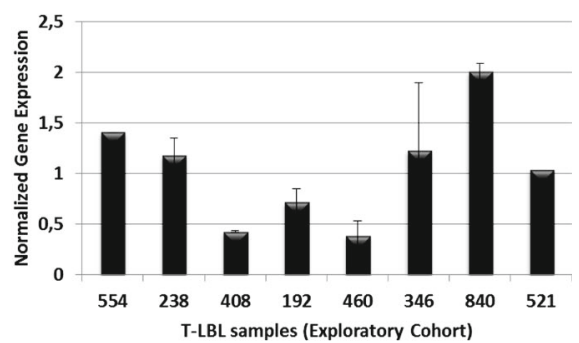

hsa-miR-25-3p

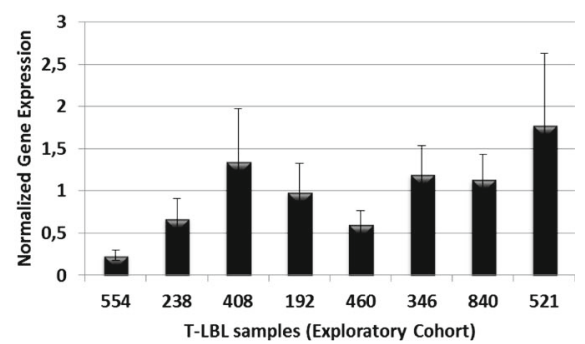

Fig. 6 Differential expression of miRNAs regulating CDKN1C, E2F1 and TP53 expression by interaction with its 3'UTR. Transcriptional levels of hsa-miR-200a-3p, hsa-miR-203, hsa-miR-205-5p, hsa-miR-221-3p, hsa-miR-222-3p, hsa-miR-25-3p and in human T-LBLs were measured using qRT-PCR assay. Relative expression values were calculated as the mRNA amount of each gene relative to miR-SNORD48 (used as reference) and normalized to the relative expression of normal control samples (foetal thymuses). Each bar represents the mean \pm SD of three independent experiments. Differences in expression values were statistically significant $(p<0.05)$

full length TAp $53 \alpha$ protein isoforms (Figs. 1 and 2). It has been demonstrated that $\Delta 133 p 53 \alpha$ does not exclusively function in a dominant-negative manner toward TAp53 $\alpha$, the full-length TP53 isoform [49], but it also inhibits TP53-dependent apoptosis [50]. Finally, two tumours (192 and 521) showed increased amounts of the TP53 $\beta$ transcript, which encodes a C-terminal truncated protein that downplay TP53 capacity to induce apoptosis $[9,51]$. These changes in the expression levels of full length and shorter isoforms may be sustained, at least in part, by deregulation of 17 miRNAs, with particular reference to miR-200a-3p and miR-375 that exhibited very high levels of downregulation in all samples in the exploratory cohort (Figs. 5 and 6).

But impairment of the TP53 function could be also attributed to the overrepresentation of the arg72 allele in our sample series (Fig. 2). It is known that the TP53 gene is not only frequently mutated in human tumours 
a

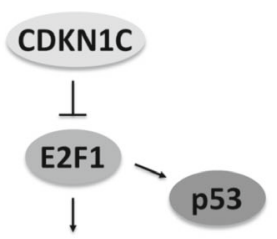

Normal thymocyte development

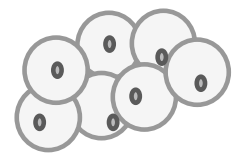

b

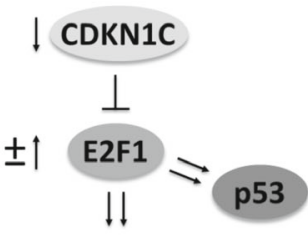

Proliferation

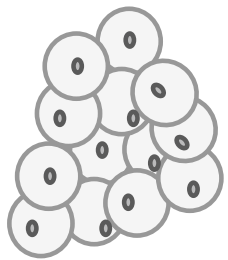

C

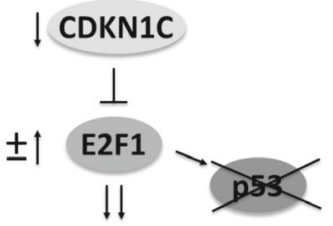

T-cell lymphoblastic neoplasia development

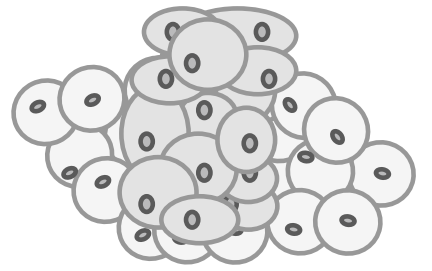

Fig. 7 CDKN1C-E2F1-TP53 axis in T-LBL development. Having in mind the consequences of alterations in these genes in mouse models [11, 34], T-LBLs in our samples series might be classified into different categories. a In normal cells CDKN1C regulates E2F1 to control the expression of E2F target genes and the activity of TP53 during thymocyte development. b Decreasing of CDKN1C (due to epigenetic mechanisms and/or upregulation of specific miRNAs) could lead to proliferation, that may be favoured by overexpression of E2F1 (through downregulation of specific miRNAs). c The additional impairment of TP53 (by the combined effect of inactivating mutations, differential expression of isoforms, and/or deregulation of specific miRNAs) should lead T-cell lymphoblastic neoplasia development

[7], but it also contains several functional polymorphisms, being by far the most common a proline (Pro) to arginine (Arg) change at codon 72 in the TP53 protein [10]. Several studies have reported preferential retention of arg72 allele in squamous cell carcinomas of the vulva [52], head and neck [53], and esophagus [54]. Considering tumour tissue DNA, Schneider-Stock et al. [55] found a significantly higher frequency of the $\arg 72$ allele in colorectal tumours and reported that the presence of this allele correlates with the malignant potential of the tumour. Similar results were also reported in urinary tract cancers [56] and lung cancer [57]. The arg72 allele was also related with increased risk for bladder cancer [58].

\section{Conclusions}

Our results indicate the existence of a CDKN1C-E2F1TP53 axis that is disrupted in a significant fraction of human T-LBLs. If, as expected, the consequences of the deregulation of the CDKN1C-E2F1-TP53 axis were the same as those experimentally demonstrated in mouse models, deregulation of this axis in human T-LBL might serve as a biomarker to predict the aggressiveness of TLBL development as depicted in Fig. 7. Furthermore, these findings would provide the basis to the development of potential therapeutic strategies based on the use of microRNAs (mimics or antagomirs) to target $C D K N 1 C$ or E2F1 deregulation that allow to rescue normal thymocyte differentiation and normal levels of thymocytes proliferation in patients with T-LBL. Blocking E2F1 expression by RNA interference might represent a promising therapeutic approach in this type of tumours. Future studies with new samples series of T-LBL should be done to be sure that the differences detected at the mRNA level translate into the protein level, and to confirm that the deregulation of this axis in human samples is really predictive of clinical outcome.

\section{Additional files}

Additional file 1: Table S1. Characterization of the human sample collection in the exploratory cohort. Lymphomas were diagnosed (see Characterization column) according to World Health Organization Classification of Hematological Malignancies and recommendations from the European. (PDF $205 \mathrm{~kb}$ )

Additional file 2: Figure S1. Gaussian Kernel Density plot of miRGate Agreement Score of the miRNAs identified associated with the CDKN1C, E2F1 and TP53 genes. (PDF $283 \mathrm{~kb}$ )

Additional file 3: Figure S2. Gaussian Kernel Density Plot of the red counts for the miRNAs deregulated in any sample. (PDF $1208 \mathrm{~kb}$ )

Additional file 4: Table S2. Description of primers used in qRT-PCR, Targeted gene deep sequencing, Sanger sequencing and Methylation-Specific PCR. (PDF 95 kb)

Additional file 5: Table S3. Differential expression of mRNA between tumours and controls in the exploratory cohort by RNA-Seq. (PDF 77 kb) Additional file 6: Table S4. Relative expression of CDKN1C, E2F1 and TP53 analyzed by qrtRT-PCR. (PDF $88 \mathrm{~kb}$ )

Additional file 7: Table S5. Complete list of genetic variants for TP53 gene determined by targeted deep sequencing in the T-LBL samples. (PDF $96 \mathrm{~kb}$ )

Additional file 8: Table S6. MicroRNA regulation of CDKN1C, E2F1 and TP53 genes in T-TLBLs of the exploratory cohort by RNA-Seq. (PDF $78 \mathrm{~kb}$ )

Additional file 9: Table S7. Validation analysis by qRT-PCR of those miRNA significantly deregulated according with RNA-Seq analysis in the exploratory and extended cohort of T-LBL samples. (PDF 81 kb) 


\section{Abbreviations}

AML: Acute myeloid leukaemia; DN3: Double-Negative 3; DN4: DoubleNegative 4; FC: Fold-change; FDR: False discovery rate; GEO: Gene Expression Omnibus; KvDMR1: Kv-Differentially Methylated Region 1;

miRNAs: microRNAs; MTI: miRNA-target interactions; NSG: Next-Generation Sequencing; qRT-PCR: Quantitative reverse transcription polymerase chain reaction),; RIN: RNA Integrity Numbers; RNA-Seq: Massive RNA-sequencing; RT-PCR: Reverse transcription polymerase chain reaction; T-ALL: T-cell acute lymphoblastic leukaemia; T-LBL: T-cell lymphoblastic lymphomas

\section{Acknowledgements}

The authors would like to thank Mario González-Sánchez and Javier González-Palacios ("Bioinformatics and Research Group in Genetic and Environmental Epidemiology", ISCIII) for their technical support. We thank all patients who were willing to donate their samples-without their support the research work would not be possible.

\section{Funding}

The authors would like to thank the Spanish Ministry of Economy and Competitiveness (SAF2015-70561-R; MINECO/FEDER, EU) and the Autonomous Community of Madrid, Spain (B2017/BMD-3778; LINFOMAS-CM) for funding this work. Institutional grants from the Fundación Ramón Areces and Banco de Santander are also acknowledged. ORCID codes: 0000-00034520-6785 to JFP and 0000-0002-4168-6251 to JS. The funding body did not play any role in the study design, collection, analysis, and interpretation of data and in writing the manuscript.

\section{Availability of data and materials}

Raw sequencing data and transcripts expression quantification is available as a superseries in GEO (Gene Expression Omnibus) under the following ID: GSE109234. The remaining datasets supporting the conclusions of this article are included within the article and its Additional files.

\section{Authors' contributions}

PLN, PFN and CVL developed the concepts, designed the experiments and contributed to the writing of the manuscript. PLN performed epigenetic experiments and analysis. PLN and CVL quantified gene expression. PFN conducted all the bioinformatics analyses. MVM, MACF, LGS and IS performed experiments. OGC, JLLL, PLI, MP and MM all read and revising the final manuscript critically. JF and JS directed the study, analyzed the results and wrote the manuscript. All authors have read and approved the final manuscript.

\section{Ethics approval and consent to participate}

The study was conducted in accordance with the Declaration of Helsinki and the Spanish legislation for the use of archived tissue specimens and associated clinical information. The clinical data were retrieved, and the histological samples were collected and analysed with the endorsement of the Madrid Autonomous University Research Ethics Committee (reference CEl: 70-1260). All the specimens were from Spanish Hospital Biobanks Network (RetBioH; www.redbiobancos.es). Biobanks authorized and inspected by National Supervisory Authority for Welfare and Health can provide human specimens collected during diagnostic procedures and associated clinical information for research purposes based on the biobank's scientific board review. Personal data will be collected, processed and stored adhering at all times to the obligation of maintaining confidentiality, in accordance with current legislation regarding the protection of personal data (Informed consent form: http://www.redbiobancos.es/DownloadHandler. ashx?f=HIP_Cl_RNBB_2012_aprobado_ING.pdf\&s=-1\&p=-1\&d=319). Identification of the biological samples of the Biobank will be subjected to a coding process. Each sample is assigned an identification code. Lymphomas were diagnosed according to World Health Organization Classification of Hematological Malignancies and recommendations from the European childhood lymphoma pathology panel.

\section{Consent for publication}

Not applicable.

\section{Competing interests}

The authors declare that they have no competing interests.

\section{Publisher's Note}

Springer Nature remains neutral with regard to jurisdictional claims in published maps and institutional affiliations.

\section{Author details}

${ }^{1}$ Department of Cellular Biology and Immunology, Severo Ochoa Molecular Biology Center (CBMSO), CSIC-Madrid Autonomous University, 28049 Madrid, Spain. ${ }^{2}$ Institute of Health Research, Jiménez Díaz Foundation, Madrid, Spain. ${ }^{3}$ Consortium for Biomedical Research in Rare Diseases (CIBERER), Carlos III Institute of Health, Madrid, Spain. ${ }^{4}$ Cancer and Environmental Epidemiology Unit, National Center for Epidemiology, Carlos III Institute of Health, Madrid, Spain. ${ }^{5}$ Consortium for Biomedical Research in Epidemiology and Public Health (CIBERESP), Madrid, Spain. 'Bioinformatics Unit, Structural Biology and Biocomputing Programme, Spanish National Cancer Research Center (CNIO), Madrid, Spain. ${ }^{7}$ Cell Division and Cancer Group, Molecular Oncology Programme, Spanish National Cancer Research Centre (CNIO), Madrid, Spain.

Received: 26 June 2017 Accepted: 26 March 2018

Published online: 16 April 2018

\section{References}

1. de Leval L, Bisig B, Thielen C, Boniver J, Gaulard P. Molecular classification of T-cell lymphomas. Crit Rev Oncol Hematol. 2009;72(2):125-43.

2. Belver $\mathrm{L}$, Ferrando $\mathrm{A}$. The genetics and mechanisms of $\mathrm{T}$ cell acute lymphoblastic leukaemia. Nat Rev Cancer. 2016;16(8):494-507.

3. Bonn BR, Krieger D, Burkhardt B. Cell cycle regulatory molecular profiles of pediatric T-cell lymphoblastic leukemia and lymphoma. Leuk Lymphoma. 2012;53(4):557-68.

4. Besson A, Dowdy SF, Roberts JM. CDK inhibitors: cell cycle regulators and beyond. Dev Cell. 2008;14(2):159-69.

5. Guo H, LV Y, Tian T, Hu TH, Wang WJ, Sui X, Jiang L, Ruan ZP, Nan KJ. Downregulation of p57 accelerates the growth and invasion of hepatocellular carcinoma. Carcinogenesis. 2011;32(12):1897-904.

6. Chen HZ, Tsai SY, Leone G. Emerging roles of E2Fs in cancer: an exit from cell cycle control. Nat Rev Cancer. 2009;9(11):785-97.

7. Olivier M, Hollstein M, Hainaut P. TP53 mutations in human cancers: origins, consequences, and clinical use. Cold Spring Harb Perspect Biol. 2010;2(1): a001008.

8. Liontos M, Niforou K, Velimezi G, Vougas K, Evangelou K, Apostolopoulou K, Vrtel R, Damalas A, Kontovazenitis P, Kotsinas A, et al. Modulation of the E2F1driven cancer cell fate by the DNA damage response machinery and potential novel E2F1 targets in osteosarcomas. Am J Pathol. 2009;175(1):376-91.

9. Khoury MP, Bourdon JC. The isoforms of the p53 protein. Cold Spring Harb Perspect Biol. 2010;2(3):a000927.

10. Bourdon JC. p53 and its isoforms in cancer. Br J Cancer. 2007;97(3):277-82.

11. Matsumoto A, Takeishi S, Nakayama Kl. p57 regulates T-cell development and prevents lymphomagenesis by balancing p53 activity and pre-TCR signaling. Blood. 2014;123(22):3429-39.

12. Oschlies I, Burkhardt B, Chassagne-Clement C, d'Amore ES, Hansson U, Hebeda K, Mc Carthy K, Kodet R, Maldyk J, Mullauer L, et al. Diagnosis and immunophenotype of 188 pediatric lymphoblastic lymphomas treated within a randomized prospective trial: experiences and preliminary recommendations from the European childhood lymphoma pathology panel. Am J Surg Pathol. 2011;35(6):836-44.

13. WHO Classification of Tumours of Haematopoietic and Lymphoid Tissues. WHO Classification of Tumours, 4th Edition, Volume 2. Edited by Swerdlow SH, Campo E, Harris NL, Jaffe ES, Pileri SA, Stein H, Thiele J, Vardiman JW. IARC (International Agency for Research on Cancer) publications; 2008.

14. Trapnell C, Roberts A, Goff L, Pertea G, Kim D, Kelley DR, Pimentel H, Salzberg SL, Rinn JL, Pachter L. Differential gene and transcript expression analysis of RNA-seq experiments with TopHat and cufflinks. Nat Protoc. 2012;7(3):562-78.

15. Langmead B, Trapnell C, Pop M, Salzberg SL. Ultrafast and memory-efficient alignment of short DNA sequences to the human genome. Genome Biol. 2009;10(3):R25.

16. Li H, Handsaker B, Wysoker A, Fennell T, Ruan J, Homer N, Marth G, Abecasis $\mathrm{G}$, Durbin R. The sequence alignment/map format and SAMtools. Bioinformatics. 2009;25(16):2078-9.

17. Yates A, Akanni W, Amode MR, Barrell D, Billis K, Carvalho-Silva D, Cummins C, Clapham P, Fitzgerald S, Gil L, et al. Ensembl 2016. Nucleic Acids Res. 2016;44(D1):D710-6. 
18. Martin M. Cutadapt removes adapter sequences from high-throughput sequencing reads. EMBnet J. 2011;17(1):3.

19. Anders S, Pyl PT, Huber W. HTSeq-a Python framework to work with highthroughput sequencing data. Bioinformatics. 2015;31(2):166-9.

20. Kozomara A, Griffiths-Jones S. miRBase: annotating high confidence microRNAs using deep sequencing data. Nucleic Acids Res. 2014; 42(Database issue):D68-73.

21. Anders S, McCarthy DJ, Chen Y, Okoniewski M, Smyth GK, Huber W, Robinson MD. Count-based differential expression analysis of RNA sequencing data using R and Bioconductor. Nat Protoc. 2013;8(9):1765-86.

22. Livak KJ, Schmittgen TD. Analysis of relative gene expression data using real-time quantitative PCR and the 2(-Delta Delta C(T)) method. Methods. 2001;25(4):402-8.

23. Chou CH, Chang NW, Shrestha S, Hsu SD, Lin YL, Lee WH, Yang CD, Hong HC, Wei TY, Tu SJ, et al. miRTarBase 2016: updates to the experimentally validated miRNA-target interactions database. Nucleic Acids Res. 2016; 44(D1):D239-47.

24. Andres-Leon E, Gonzalez Pena D, Gomez-Lopez G, Pisano DG. miRGate: a curated database of human, mouse and rat miRNA-mRNA targets. Database (Oxford). 2015;2015:bav035.

25. Mullokandov G, Baccarini A, Ruzo A, Jayaprakash AD, Tung N, Israelow B, Evans MJ, Sachidanandam R, Brown BD. High-throughput assessment of microRNA activity and function using microRNA sensor and decoy libraries. Nat Methods. 2012;9(8):840-6.

26. Bouaoun L, Sonkin D, Ardin M, Hollstein M, Byrnes G, Zavadil J, Olivier M. TP53 variations in human cancers: new lessons from the IARC TP53 database and genomics data. Hum Mutat. 2016;37(9):865-76.

27. Matsuoka S, Thompson JS, Edwards MC, Bartletta JM, Grundy P, Kalikin LM, Harper JW, Elledge SJ, Feinberg AP. Imprinting of the gene encoding a human cyclin-dependent kinase inhibitor, p57KIP2, on chromosome 11 p15. Proc Natl Acad Sci U S A. 1996;93(7):3026-30.

28. Negrini M, Ferracin M, Sabbioni S, Croce CM. MicroRNAs in human cancer: from research to therapy. J Cell Sci. 2007;120(Pt 11):1833-40.

29. Bueno MJ, Malumbres M. MicroRNAs and the cell cycle. Biochim Biophys Acta. 2011;1812(5):592-601.

30. Jin RJ, Lho Y, Wang Y, Ao M, Revelo MP, Hayward SW, Wills ML, Logan SK Zhang P, Matusik RJ. Down-regulation of p57Kip2 induces prostate cancer in the mouse. Cancer Res. 2008;68(10):3601-8.

31. Schwarze SR, Shi Y, Fu VX, Watson PA, Jarrard DF. Role of cyclin-dependent kinase inhibitors in the growth arrest at senescence in human prostate epithelial and uroepithelial cells. Oncogene. 2001;20(57):8184-92.

32. Lu L, Qiu J, Liu S, Luo W. Vitamin D3 analogue EB1089 inhibits the proliferation of human laryngeal squamous carcinoma cells via p57. Mol Cancer Ther. 2008;7(5):1268-74.

33. Dos Reis VL, Pujiz RS, Strauss BE, Krieger JE. Knockdown of E2f1 by RNA interference impairs proliferation of rat cells in vitro. Genet Mol Biol. 2010; 33(1):17-22

34. Jayapal SR, Kaldis P. p57(Kip2) regulates T-cell development and lymphoma. Blood. 2014;123(22):3370-1.

35. de Sousa AR, Penalva LO, Marcotte EM, Vogel C. Global signatures of protein and mRNA expression levels. Mol BioSyst. 2009:5(12):1512-26.

36. Koussounadis A, Langdon SP, Um IH, Harrison DJ, Smith VA. Relationship between differentially expressed mRNA and mRNA-protein correlations in a xenograft model system. Sci Rep. 2015;5:10775.

37. Pateras IS, Apostolopoulou K, Niforou K, Kotsinas A, Gorgoulis VG. p57KIP2: "KIP"ing the cell under control. Mol Cancer Res. 2009;7(12):1902-19.

38. Mancini-DiNardo D, Steele SJ, Ingram RS, Tilghman SM. A differentially methylated region within the gene Kcnq1 functions as an imprinted promoter and silencer. Hum Mol Genet. 2003;12(3):283-94.

39. Li Y, Nagai H, Ohno T, Yuge M, Hatano S, Ito E, Mori N, Saito H, Kinoshita T. Aberrant DNA methylation of p57(KIP2) gene in the promoter region in lymphoid malignancies of B-cell phenotype. Blood. 2002;100(7):2572-7.

40. Shen L, Toyota M, Kondo Y, Obata T, Daniel S, Pierce S, Imai K, Kantarjian HM, Issa JP, Garcia-Manero G. Aberrant DNA methylation of p57KIP2 identifies a cell-cycle regulatory pathway with prognostic impact in adult acute lymphocytic leukemia. Blood. 2003;101(10):4131-6.

41. Kikuchi T, Toyota M, Itoh F, Suzuki H, Obata T, Yamamoto H, Kakiuchi H, Kusano M, Issa JP, Tokino T, et al. Inactivation of p57KIP2 by regional promoter hypermethylation and histone deacetylation in human tumors. Oncogene. 2002;21(17):2741-9.
42. Yang X, Karuturi RK, Sun F, Aau M, Yu K, Shao R, Miller LD, Tan PB, Yu Q. CDKN1C (p57) is a direct target of EZH2 and suppressed by multiple epigenetic mechanisms in breast cancer cells. PLoS One. 2009;4(4):e5011.

43. Fornari F, Gramantieri L, Ferracin M, Veronese A, Sabbioni S, Calin GA, Grazi GL, Giovannini C, Croce CM, Bolondi L, et al. MiR-221 controls CDKN1C/p57 and CDKN1B/p27 expression in human hepatocellular carcinoma. Oncogene. 2008;27(43):5651-61.

44. Pulikkan JA, Dengler V, Peramangalam PS, Peer Zada AA, Muller-Tidow C, Bohlander SK, Tenen DG, Behre G. Cell-cycle regulator E2F1 and microRNA223 comprise an autoregulatory negative feedback loop in acute myeloid leukemia. Blood. 2010;115(9):1768-78.

45. Kim YK, Yu J, Han TS, Park SY, Namkoong B, Kim DH, Hur K, Yoo MW, Lee HJ, Yang HK, et al. Functional links between clustered microRNAs: suppression of cell-cycle inhibitors by microRNA clusters in gastric cancer. Nucleic Acids Res. 2009;37(5):1672-81.

46. Burkhart DL, Sage J. Cellular mechanisms of tumour suppression by the retinoblastoma gene. Nat Rev Cancer. 2008;8(9):671-82.

47. Polager S, Ginsberg D. E2F - at the crossroads of life and death. Trends Cell Biol. 2008;18(11):528-35.

48. Dar AA, Majid S, de Semir D, Nosrati M, Bezrookove V. Kashani-Sabet M: miRNA-205 suppresses melanoma cell proliferation and induces senescence via regulation of E2F1 protein. J Biol Chem. 2011;286(19):16606-14.

49. Bourdon JC, Fernandes K, Murray-Zmijewski F, Liu G, Diot A, Xirodimas DP, Saville MK, Lane DP. p53 isoforms can regulate p53 transcriptional activity. Genes Dev. 2005;19(18):2122-37.

50. Aoubala M, Murray-Zmijewski F, Khoury MP, Fernandes K, Perrier S, Bernard $H$, Prats AC, Lane DP, Bourdon JC. p53 directly transactivates Delta133p53alpha, regulating cell fate outcome in response to DNA damage. Cell Death Differ. 2011;18(2):248-58.

51. Olivier M, Petitjean A, Marcel V, Petre A, Mounawar M, Plymoth A, de Fromentel CC, Hainaut P. Recent advances in p53 research: an interdisciplinary perspective. Cancer Gene Ther. 2009;16(1):1-12.

52. Brooks LA, Tidy JA, Gusterson B, Hiller L, O'Nions J, Gasco M, Marin MC, Farrell PJ, Kaelin WG Jr, Crook T. Preferential retention of codon 72 arginine p53 in squamous cell carcinomas of the vulva occurs in cancers positive and negative for human papillomavirus. Cancer Res. 2000;60(24):6875-7.

53. Schneider-Stock R, Mawrin C, Motsch C, Boltze C, Peters B, Hartig R, Buhtz P, Giers A, Rohrbeck A, Freigang B, et al. Retention of the arginine allele in codon 72 of the p53 gene correlates with poor apoptosis in head and neck cancer. Am J Pathol. 2004;164(4):1233-41.

54. Kawaguchi H, Ohno S, Araki K, Miyazaki M, Saeki H, Watanabe M, Tanaka S, Sugimachi K. p53 polymorphism in human papillomavirus-associated esophageal cancer. Cancer Res. 2000;60(11):2753-5.

55. Schneider-Stock R, Boltze C, Peters B, Szibor R, Landt O, Meyer F, Roessner A. Selective loss of codon 72 proline p53 and frequent mutational inactivation of the retained arginine allele in colorectal cancer. Neoplasia. 2004;6(5):529-35.

56. Furihata M, Takeuchi T, Matsumoto M, Kurabayashi A, Ohtsuki Y, Terao N, Kuwahara M, Shuin T. p53 mutation arising in Arg72 allele in the tumorigenesis and development of carcinoma of the urinary tract. Clin Cancer Res. 2002:8(5):1192-5.

57. Papadakis ED, Soulitzis N, Spandidos DA. Association of p53 codon 72 polymorphism with advanced lung cancer: the Arg allele is preferentially retained in tumours arising in Arg/pro germline heterozygotes. $\mathrm{Br} J$ Cancer. 2002;87(9):1013-8.

58. Soulitzis N, Sourvinos G, Dokianakis DN, Spandidos DA. p53 codon 72 polymorphism and its association with bladder cancer. Cancer Lett. 2002; 179(2):175-83. 